\title{
Mutations in NEBL encoding the cardiac Z-disk protein nebulette are associated with various cardiomyopathies
}

Andreas Perrot ${ }^{1}$, Pavol Tomasov², Eric Villard ${ }^{3}$, Reka Faludi ${ }^{4}$, Paola Melacini ${ }^{5}$, Janine Lossie ${ }^{1}$, Nadine Lohmann ${ }^{1}$, Pascale Richard ${ }^{6}$, Marzia De Bortoli ${ }^{5}$, Annalisa Angelini ${ }^{5}$, Akos Varga-Szemes ${ }^{4}$, Silke R. Sperling' ${ }^{1}$, Tamás Simor ${ }^{4}$, Josef Veselka², Cemil Özcelik ${ }^{1,7}$, Philippe Charron ${ }^{3,8}$

${ }^{1}$ Charité-Universitätsmedizin Berlin, Cardiovascular Genetics, Experimental and Clinical Research Center, Berlin, Germany

${ }^{2}$ Department of Cardiology, $2^{\text {nd }}$ Medical School, Charles University, University Hospital Motol, Prague, Czech Republic

${ }^{3}$ AP-HP, Département de Génétique et Département de Cardiologie et Inserm UMR

1166, Hopital Pitié-Salpêtrière, Paris, France

${ }^{4}$ Heart Institute, Faculty of Medicine, University of Pécs, Pécs, Hungary

${ }^{5}$ Department of Cardiac, Thoracic and Vascular Sciences, University of Padova,

Padova, Italy

${ }^{6}$ Assistance Publique-Hôpitaux de Paris, Groupe Hospitalier Pitié-Salpêtrière,

UF Cardiogénétique et Myogénétique, Service de Biochimie Métabolique, Paris, France ${ }^{7}$ Knappschaftskrankenhaus Recklinghausen, Medizinischen Klinik I Kardiologie,

Gastroenterologie und Diabetologie, Recklinghausen, Germany

${ }^{8}$ Université de Versailles Saint Quentin en Yvelines, Versailles, France

Submitted: 4 February 2015

Accepted: 29 April 2015

Arch Med Sci 2016; 12, 2: 263-278

DOI: 10.5114 /aoms.2016.59250

Copyright (c) 2016 Termedia \& Banach

\section{Abstract}

Introduction: Transgenic mice overexpressing mutated NEBL, encoding the cardiac-specific Z-disk protein nebulette, develop severe cardiac phenotypes. Since cardiomyopathies are commonly familial and because mutations in a single gene may result in variable phenotypes, we tested the hypothesis that NEBL mutations are associated with cardiomyopathy.

Material and methods: We analyzed 389 patients, including cohorts of patients with dilated cardiomyopathy (DCM), hypertrophic cardiomyopathy (HCM), and left ventricular non-compaction cardiomyopathy (LVNC). The 28 coding exons of the NEBL gene were sequenced. Further bioinformatic analysis was used to distinguish variants.

Results: In total, we identified six very rare heterozygous missense mutations in NEBL in 7 different patients (frequency $1.8 \%$ ) in highly conserved codons. The mutations were not detectable in 320 Caucasian sex-matched unrelated individuals without cardiomyopathy and 192 Caucasian sex-matched blood donors without heart disease. Known cardiomyopathy genes were excluded in these patients. The mutations p.H171R and p.1652L were found in $2 \mathrm{HCM}$ patients. Further, p.Q581R and p.S747L were detected in 2 DCM patients, while the mutation p.A175T was identified independently in two unrelated patients with DCM. One LVNC patient carried the mutation p.P916L. All HCM and DCM related mutations were located in the nebulin-like repeats, domains responsible for actin binding. Interestingly, the mutation associated with LVNC was located in the C-terminal serine-rich linker region.

Conclusions: Our data suggest that NEBL mutations may cause various cardiomyopathies. We herein describe the first NEBL mutations in HCM and LVNC. Our findings underline the notion that the cardiomyopathies are true allelic diseases.

Key words: cardiomyopathy, hypertrophic, dilated, non-compaction, genetics.

\author{
Corresponding author: \\ Andreas Perrot MSc \\ Cardiovascular Genetics \\ Charité-Universitätsmedizin \\ Experimental and Clinical \\ Research Center (ECRC) \\ Lindenberger Weg 80 \\ 13125 Berlin, Germany \\ Phone: +4930450540168 \\ E-mail: \\ andreas.perrot@charite.de
}


A. Perrot, P. Tomasov, E. Villard, R. Faludi, P. Melacini, J. Lossie, N. Lohmann, P. Richard, M. De Bortoli, A. Angelini, A. Varga-Szemes, S.R. Sperling, T. Simor, J. Veselka, C. Özcelik, P. Charron

\section{Introduction}

Cardiomyopathies are heterogeneous heart muscle diseases that commonly have a genetic cause $[1,2]$. Whereas hypertrophic cardiomyopathy (HCM) and left ventricular non-compaction cardiomyopathy (LVNC) are classified as genetic forms according to the American Heart Association, dilated cardiomyopathy (DCM) is familial in about one third of patients. The heterogeneous nature of the cardiomyopathies can be seen at the clinical and the genetic level $[1,2]$. In HCM, the known disease genes may account for genetic causation in only about $50-60 \%$ of patients $[1,2]$. Presumably additional disease genes exist. Recent studies also changed our earlier view of HCM as a solely sarcomeric and DCM as a solely cytoskeletal disorder. It has been shown that considerable overlap of disease-causing genes exists between the main phenotypically distinct forms of cardiomyopathy [3, 4]. Moreover, mutations in certain genes may cause very variable phenotypes $[3,4]$.

Mutations in Z-disk associated proteins have been linked to cardiomyopathy $[2,5]$. The cardiac-specific protein nebulette, encoded by $N E B L$, is a good candidate, since the protein aligns thin filaments and connects them to the Z-disk. Nebulette belongs to the nebulin family of actin-binding proteins that are important in myofibrillogenesis $[6,7]$ and assembly of the Z-line [8]. Nebulette also interacts with filamin C [9], myopalladin [10], $\alpha$-actinin 2 [7], and tropomyosin and troponin $T$ $[11,12]$. The key role of $N E B L$ is further supported by the recent generation of transgenic mice overexpressing NEBL mutations in the heart and showing severe cardiac phenotypes [13].

We therefore selected NEBL as a candidate gene in patients with cardiomyopathies and systematically analyzed the gene in three well-characterized cohorts of patients with HCM, DCM, and LVNC.

\section{Material and methods}

\section{Patients}

We studied 389 unrelated cardiomyopathy patients with HCM, DCM, or LVNC of mostly European origin. The respective local institutional review boards (IRB) in Berlin, Paris, Prague, Pecs and Padova approved the study and written informed consent was obtained from all participants. The genetic analysis was performed in Berlin (IRB vote number $A A / 3 / 01 / 32$ for genetic analysis in cardiomyopathy patients). The study protocol conforms to the ethical guidelines of the 1975 Declaration of Helsinki.

The HCM cohort comprised 217 patients. The patients were managed either in Paris (96 patients), in Padova (26 patients) or in Prague (95 pa- tients). The diagnosis of HCM was made according to the established criteria as described by us in detail previously $[14,15]$. The DCM cohort in cluded 148 patients. The patients were managed either in Paris (114 patients; 65 familial and 49 sporadic) or in Berlin (34 familial patients). Diagnosis of DCM was made according to the established criteria as used and described previously $[16,17]$. The LVNC cohort comprised 24 patients managed in Pecs. Relatives of patients with identified mutation were recruited after the initial genetic analysis and subsequently selected from their families. Diagnosis of LVNC was based on the criteria proposed by Petersen et al. [18] The control cohort A comprised 320 sex-matched unrelated individuals (European origin) without cardiomyopathy. The second control cohort B included 192 sex-matched blood donors (European origin) without heart disease. Further information about the cohorts can be found in the Supplemental methods.

\section{Genetic analysis}

Genomic DNA was isolated using standard methods. Primer pairs were used to amplify the 28 coding exons of NEBL with flanking intronic sequences according to Arimura et al. [19] based on the published sequence (GenBank accession number NM_006393.2). Polymerase chain reaction amplifications were performed using standard protocols (available on request). The amplicons were analyzed by direct sequencing using $A B I B i g$ Dye Terminator chemistry and run on an ABI 3100 Avant device (Applied Biosystems, Darmstadt, Germany) as per the manufacturer's instructions. Detected variants in a sample were confirmed at least in two independent PCRs and sequencing runs. Sequencher software version 4.8 (Gene Codes, Ann Arbor, MI, USA) or PhredPhrap/Consed free Softs was used to facilitate data analysis and mutation identification followed by visual inspection of individual sequencing traces. Genetic variants were annotated according to the cDNA and protein reference sequence (Ensembl ID ENST00000377122 and UniProtKB/Swiss-Prot O76041).

We further analyzed the detected variants by checking the presence or absence of these variants in large population-based databases (dbSNP and Exome Sequencing Project/ESP5400 dataset) and the possible impact of the amino acid substitution. Further information about the bioinformatic analysis can be found in the Supplemental Methods. Briefly, the significance of the identified variants was assessed by considering the conservation of the affected amino acid, the nature and location of the change, its rarity assessed in own controls and population-based datasets, and cosegregation in the family when available. 


\section{Results}

\section{$N E B L$ mutations}

We sequenced NEBL in 389 cardiomyopathy patients and identified 20 different genetic variants (Figure 1). Fourteen variants were detected in different frequencies (from 1\% to 31\%) and were regarded as polymorphisms (Figure $1 \mathrm{~A}$ and see also Supplemental results and Supplemental Table SI). In contrast, six variants were detected in 1 patient each: p.H171R, p.A175T, p.Q581R, p.1652L, p.S747L, and p.P916L (with the exception of p.A175L, which was detected in two unrelated patients) (Table I). These were regarded as possibly disease-associated mutations because of the following facts. All six were heterozygous missense mutations (Supplemental Figure S1); none was detectable in 640 control alleles. Two mutations were not annotated in dbSNP (p.H171R, p.Q581R); we deposited two (p.A175T, p.S747L) (Table I). The mutations p.1652L and p.P916L were subsequently annotated in dbSNP with low allelic frequencies of $0.3 \%$ and $0.08 \%$, respectively (Table I). While four mutations were absent from the ESP5400 dataset (European Americans), two (p.A175T and p.1652L) occurred at a very low frequency of $<0.01 \%$. Five amino acid substitutions altered a non-conserved residue (the exception was p.1652L). Two independent mutation analysis tools predicted damaging effects for the six mutations (either PolyPhen2 and/or Mutation Taster; see Supplemental methods).

Mutation p.P916L is located in the N-terminal serine-rich linker region; the other five mutations are located in the central nebulin-like repeats (NLRs). These are important actin-binding modules of the protein (Figure $1 \mathrm{~B}$ ). Alignment of sequences surrounding mutations from different orthologs (from chimpanzee to fish) showed very high conservation of the residue through evolution for three mutations (p.H171R, p.1652L, p.S747L) to high conservation for the other three mutations (Figure 2 B). Alignment of the 23 NLRs showed that three mutations (p.Q581R, p.1652L, p.S747L) are located in or close to the two common consensus motifs (PEIXRXK box and SDXXYK box) in these domains (Figure $2 \mathrm{~A}$ ). The amino acids glutamine (p.Q581R) and isoleucine (p.1652L) are also conserved in different nebulette NLRs.

Mutations p.H171R and p.1652L were found in 2 HCM patients. Further, the mutations p.Q581R and p.S747L were detected in 2 familial DCM patients, while the mutation p.A175T was detected

A

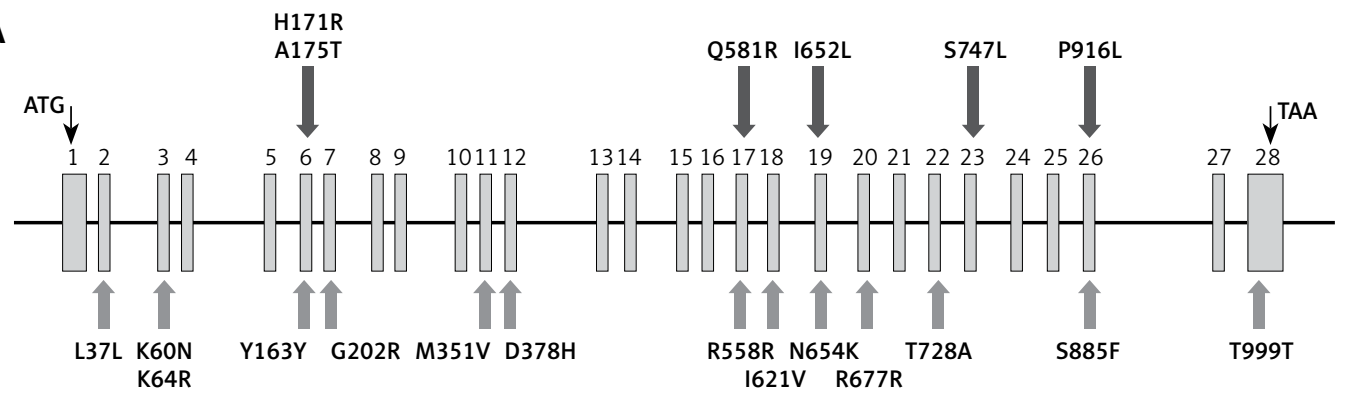

B

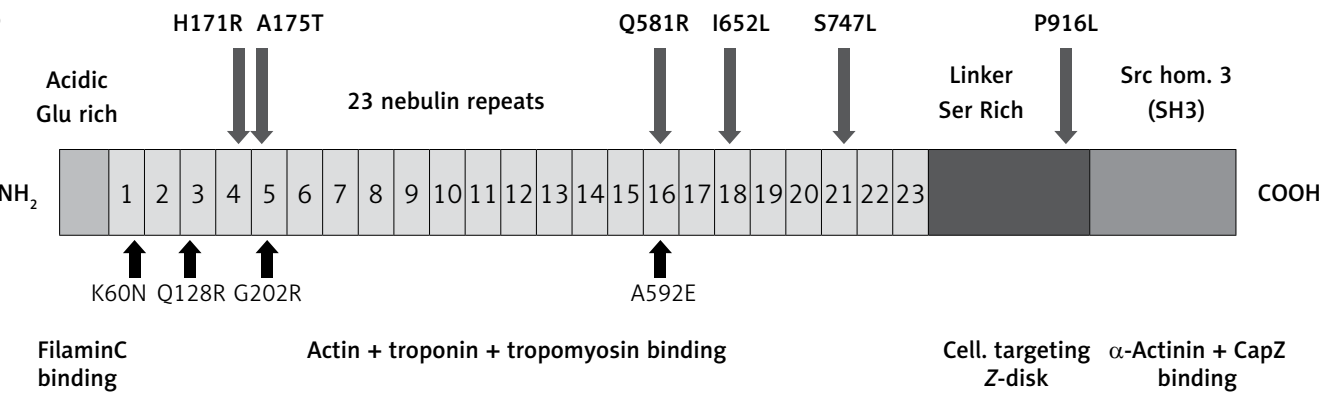

Figure 1. Localization of the mutations and other variants in the nebulette gene and protein. A - Distribution of detected variants in NEBL. In the schematic diagram, gray blocks symbolize the 28 exons of the gene. Very rare variants (possible disease-associated mutations) are indicated as black arrows above their exonic location. Other more frequent synonymous and non-synonymous variants are indicated by small gray arrows below their exonic location. B - Localization of the identified mutations in the nebulette protein. In the schematic diagram, gray blocks symbolize different protein domains. Nebulette consists of an N-terminal acidic glutamic acid-rich domain, 23 central nebulin-like repeats (NLR), a serine-rich linker domain, and a C-terminal Src homology 3 (SH3) domain. Binding partners and interactions are shown below. The six possible mutations identified by us are denoted by long black arrows above the diagram, whereas mutations identified by Purevjav et al. [13] are denoted by small black arrows below the diagram. Notably, some of the mutations are close together, e.g. affecting the same NLR in two cases (schematic representations and interactions according to $[6,8,29]$ ) 


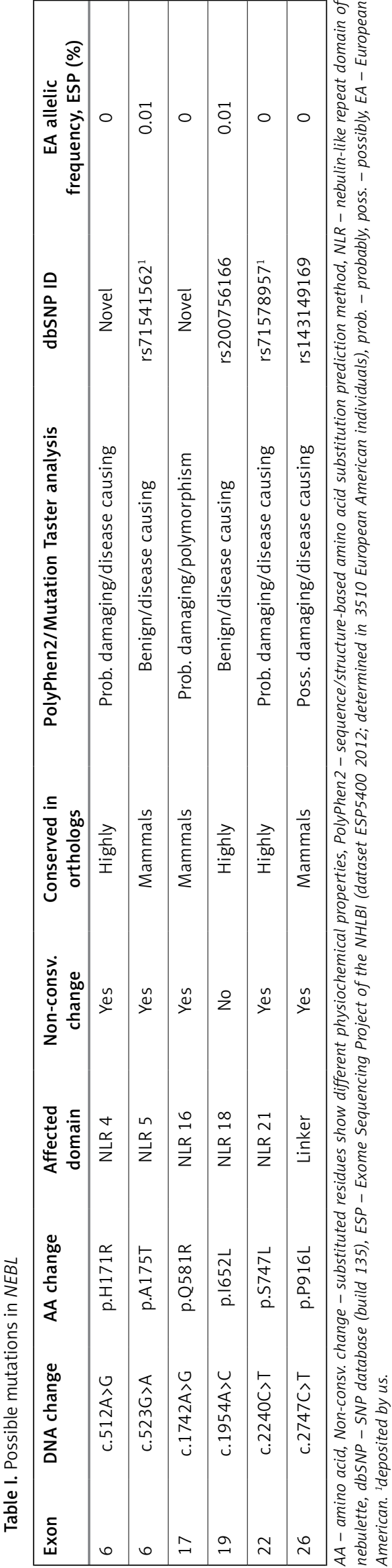

in 2 unrelated patients with DCM. One LVNC patient carried p.P916L. Overall, this occurrence has a frequency of $1.8 \%$ (7/389). Mutation screening performed previously on several other cardiomyopathy genes in these patients was negative (details see below). As commonly observed in cardiomyopathy, the small size of the families and the age-related incomplete penetrance only allowed cosegregation analysis in the families. The pedigrees are shown in alphabetical order in Figure 3. The clinical data of these patients are summarized in Table II.

\section{Clinical features in HCM}

The symptomatic male patient (proband A: II- 6 of family A) of Czech origin, carrier of mutation p.H171R, had HCM since the age of 53 years. He presented with moderate hypertrophy including an interventricular septal thickness (IVS) of $18 \mathrm{~mm}$ and posterior wall thickness (PWT) of $13 \mathrm{~mm}$. His sister (A: II-5), 56 years old, was a carrier of the mutation but showed no signs of HCM. All other family members were negative for the mutation and also showed no HCM. Individuals A: II-2, A: II-3, and A: II-4 showed IVS values of 11-12 mm; all were treated for arterial hypertension.

The Italian female patient (proband B: II-3 of family B), carrier of mutation p.1652L, was diagnosed with juvenile HCM with severe hypertrophy at the age of 14 years. She received an implantable cardioverter defibrillator (ICD) because of ventricular tachycardia (further clinical data in the Supplemental Results and Supplemental Figure S2). Her mother (B: I-2), 69 years old, carried the mutation and showed mild septal hypertrophy. But she was operated on because of calcific aortic stenosis and a bicuspid aortic valve. Her father (B: I-1), aged 74 years, demonstrated HCM with an age of onset at 60 years and later on developed hypertension but did not carry the mutation. Of note, her brother (B: II-2), aged 45 years and a non-carrier of the mutation, showed mild hypertrophy and was treated for hypertension for about 20 years. The 3 children (B: III-1, B: III-2, and B: III-3), aged from 18 to 22 years and each of whom had normal values in echocardiography, also did not carry the mutation. Neither of the above families had mutations in sarcomeric HCM genes (analyzed by DNA resequencing array by us and coworkers [20]).

\section{Clinical features in DCM}

The 47-year-old German patient (proband II-4 of family C), carrier of mutation p.A175T, had disease onset at the age of 45 years and had severe DCM with a strongly dilated left ventricle and poor LV function (EF 15\%). ECG showed atrial fibrillation as well as non-sustained ventricular tachy- 


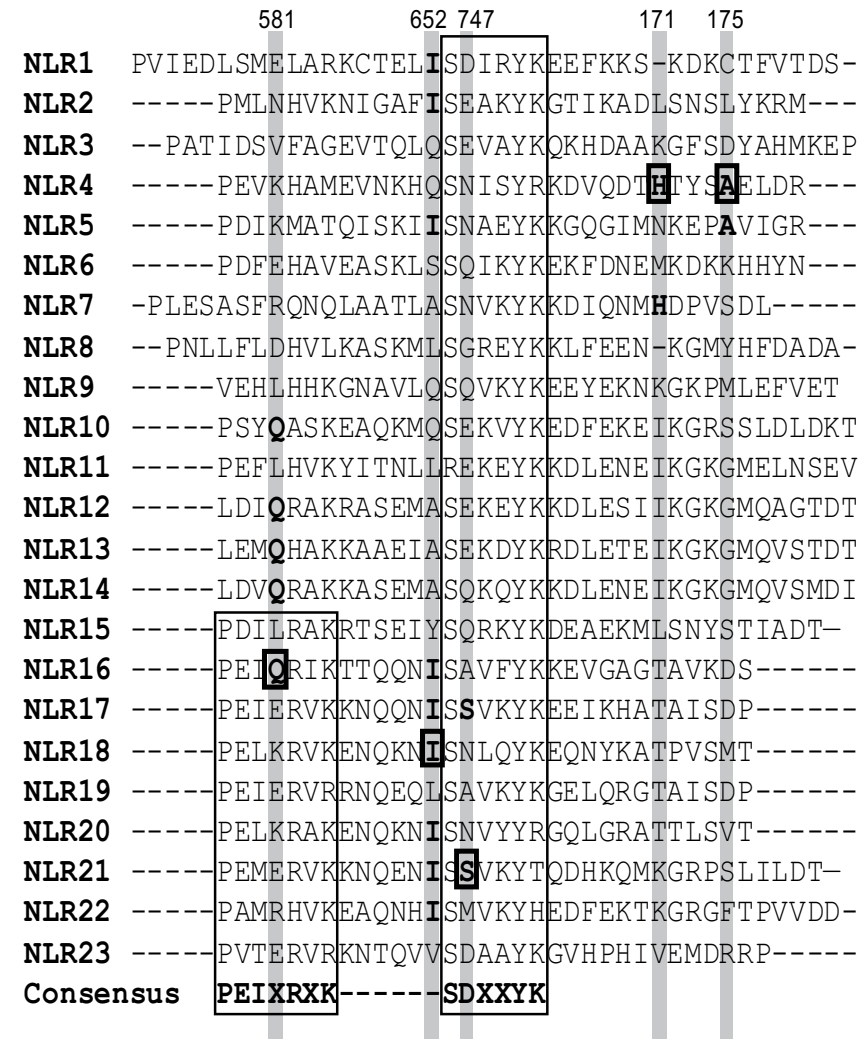

\section{B}

Codon
Human
Chimpanzee
Orang utan
Macaque
Rabbit
Panda
Horse
Cow
Dog
Mouse
Rat
Opossum
Platypus
Chicken
Zebra finch
Anole lizard
Medaka

\begin{tabular}{|c|c|}
\hline $171 \quad 175$ & 581 \\
\hline THTYSAEELD & TPEIQRIKT \\
\hline$\overline{\mathbf{H}} T Y R \overline{\mathbf{A}} E L D$ & TPEMQRIKT \\
\hline THTYSAELD & TPEIQRIKT \\
\hline AHTYSAELD & TPEIRRIKT \\
\hline HTYSAELD & TPEMQRIKT \\
\hline THTYSADLN & TPEIQRIKT \\
\hline SHMYSAELD & SPEIQRIKT \\
\hline THMYNAELD & TPEIQRRIKT \\
\hline THMYSADLN & TPEIQRIKT \\
\hline THTYTAELD & TPEIQRIKT \\
\hline THTYTAEMD & TPEIQRIKT \\
\hline NHKYNAGID & TPEIQRIKT \\
\hline VHKYTAGLD & TPEIQRIKI \\
\hline THRYTEVLN & TPEMERIKS \\
\hline THRYTEVLN & TPEMERIRS \\
\hline IHKYTEVSK & TPEIKRVKD \\
\hline LHHCNMVPD & TPEMERVRA \\
\hline
\end{tabular}

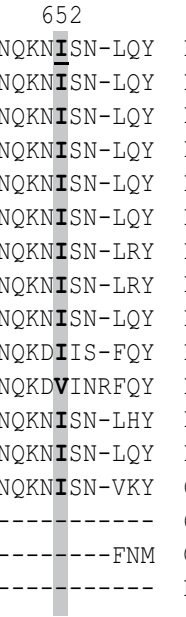

747
EISSVKYT
NISSVKYT
ENISSVKYT
EISSVKYT
EISSVKYT
EISSIKYT
EISSVKYT
ENISSVKYT
EISSVKYT
NISSVKYT
NISSVKYT
NISSVKYT
NISSVKYT
NISSIKYK
NISSIKYK
NISAVSFN
ENISSVK--

916
EVTRPDE
EITRSDE
EVTRPSDE
AVTRLSDL
ELTRPSDD
EVTRPSDE
EVTRPSDE
EVTRPSDE
EVTRPSDE
EVTRPSDE
EVTRPSDE
EITRPSDE
EVTRGSDE
------
EVTKPSNE

Figure 2. Conservation of the identified possible mutations p.H171R, p.A175T, p.Q581R, p.1652L, p.S747L, and p.P916L. A - Alignment of the 23 copies of nebulin-like repeats (NLR) present in human nebulette (according to Millevoi et al. [30]) with mutations p.H171R, p.A175T, p.Q581R, p.1652L, p.S747L (small boxes). The NLRs are modules of about 35 residues present in all members of the nebulin protein family. They are characterized by the sequence motif SDXXYK (open box). NLR 15 to 22 additionally share a conserved motif (PEIXRXK box at their N-termini). The mutations are located in or close to these consensus motifs. The amino acids glutamine (p.Q581R) and isoleucine (p.1652L) are also conserved in different nebulette NLRs (as shown by bold letters). B - Alignment of orthologs from 16 different species (from mammals and birds to fish). The altered amino acid and flanking residues are displayed in single-letter code. Bold letters mean conservation. Mutated residue in the human sequence is underlined

cardia; an ICD was implanted. His twin brother (C: II-3) is also a carrier of the mutation and awaits heart transplantation (TX) because of severe DCM. His female cousin (C: III-1) underwent TX because of DCM but was not available for genetic analysis. The proband has two male children (C: III-2, 19 years old, and C: III-3, 23 years old) who carried the mutation but were as yet disease free. His father (C: I-4) died of heart failure. The symptomatic brother (C: II-6) showed a mildly dilated heart. He was diagnosed with an alcohol-toxic form of DCM because of known longterm excessive alcohol consumption and showed a negative genotype. 
A. Perrot, P. Tomasov, E. Villard, R. Faludi, P. Melacini, J. Lossie, N. Lohmann, P. Richard, M. De Bortoli, A. Angelini, A. Varga-Szemes, S.R. Sperling, T. Simor, J. Veselka, C. Özcelik, P. Charron

Family A (HCM; p.H171R)

I

$$
\text { II }
$$<smiles>CCCCCc1ccccc1</smiles><smiles>Cc1ccc(C)c(C(C)C2CCC2)c1</smiles>

III

Family C (DCM; p.A175T)

I<smiles>Cc1ccccc1C(C)C1CCC1</smiles>

"

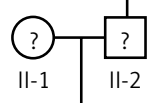

III III-1

Family E (DCM; p.A175T)

I

II

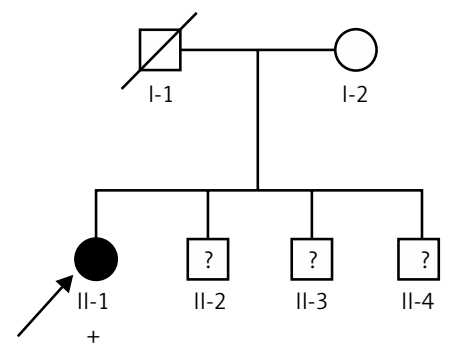

Family F (DCM; p.S747L)

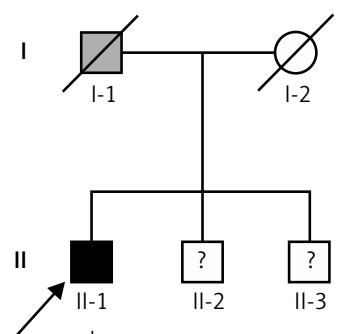

Family B (HCM; p.1652L)

I

II

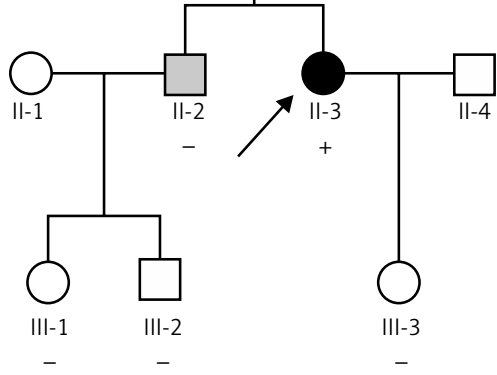

Family D (DCM; p.Q581R)

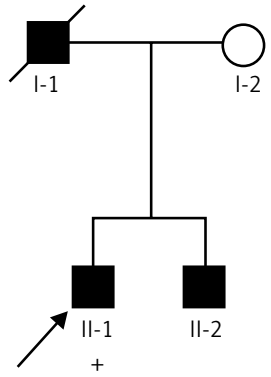

Family G (LVNC; p.P916L)

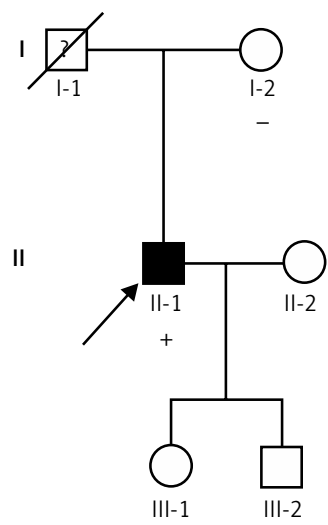

Figure 3. Pedigrees of the families. Squares indicate males, circles females. Open symbols represent unaffected subjects and solid symbols affected individuals according to clinical examinations; shaded symbols represent individuals with borderline status; question marks represent individuals with unknown status where no clinical data were available; and slanted bars represent deceased individuals. The presence or absence of an NEBL mutation is indicated by a "+" or "-" symbol, respectively. No such symbol indicates that no DNA was available for analysis. An arrow denotes the proband 
The German male patient (proband D: II-1 of family D), aged 52 years and carrier of mutation p.Q581R, had symptoms (including dizziness and dyspnea on exertion) since the age of 46 . Holter ECG showed intermittent tachycardia. He has a dilated LV and borderline LV function (48\%). His father (D: I-1) died at the age of 52 years because of DCM. His 60-year-old brother (D: II-2) awaits TX. No DNA was available from either. The disease genes LMNA, MYH7, ACTC, DES, PLN, MLP, TNNC, $T N N /$ were excluded in both probands of families $\mathrm{C}$ and $\mathrm{D}$.

The French female patient (proband E: II-1 of family E), carrier of mutation p.A175T, was diagnosed with DCM at the age of 48 years. She showed mild dilatation and diminished LV function (EF 40\%). Proband E: II-1 has a sporadic form of DCM; no other family members were affected or were available for genetic analysis.

The French male patient (proband F: II-1 of family F), carrier of mutation S747L and of Syrian origin, showed disease onset at the age of 49 years. He had severe dilatation and poor LV function with an EF of $35 \%$. His father (F: I-1) died at the age of 63 years because of heart failure. No other family members were available for clinical and genetic examination. Important DCM disease genes (LMNA, MYH7, ACTC, DES, SGCD, TNNT2, $P L N, M Y P N$, and the cardiospecific exon 16 of $V C L$ ) were excluded in both probands of family $E$ and $F$.

\section{Clinical features in LVNC}

The 47-year-old male patient of Hungarian origin (proband G: II-1 of family G), carrier of mutation p.P916L, has been treated because of DCM and heart failure since the age of 37 years. Echocardiography suggested the presence of hypertrabecularization in the apical region of the left ventricle. Cardiac magnetic resonance imaging (MRI) confirmed LVNC as described in Supplemental results and Supplemental Figure S3. His 67-year-old mother (G: I-2) and his two children (G: III-1 and G: III-2), 25 and 19 years old, respectively, were negative for the mutation and were unaffected as shown by echo and/or MRI. The proband G: II-1 had been analyzed previously for mutations in 12 known cardiomyopathy genes as described by us [20], and no mutation was found in any of these genes.

\section{Discussion}

We identified six novel heterozygous missense mutations in NEBL in seven different patients and their families. We excluded known cardiomyopathy genes in these patients, particularly the most frequent HCM disease genes [14, 20]. Overall, our data are highly suggestive for disease causation,

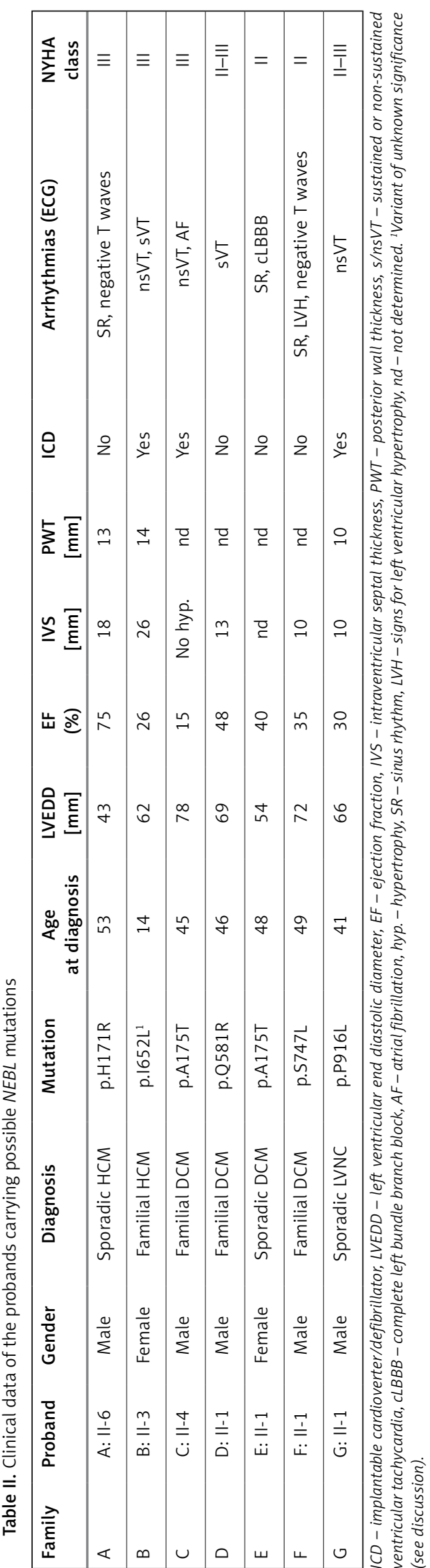


although without definite proof because of the small size of most families. The presence of six different mutations in seven unrelated cardiomyopathy patients makes the possible explanation of rare polymorphisms highly unlikely. This in terpretation is confirmed by the absence of the mutations in a high number of ethnically matched control individuals. Despite the very low frequency of the mutations ( $<1 / 1000$ alleles), we detected the 175 variant in two independent patients from Germany and France. Exome Sequencing Project (ESP) and dbSNP datasets confirmed the rarity/ novelty of all mutations, which is a major criterion for pathogenicity in cardiomyopathies with so many "private" mutations $[1,2,4]$. Norton et al. proposed using the ESP dataset as a new resource for guidance in DCM patients [21]. They found an allele frequency of $0.04 \%$ as a conservative cutoff point for pathogenic mutations based on their bioinformatic analysis of DCM mutations against the ESP dataset. Using the same approach for $N E B L$, all mutations found by us are below that threshold, confirming their extreme rarity (Table I).

We are aware that a non-synonymous mutation causing a genetic disease increases in evolutionarily highly conserved sites [22]. The affected amino acids of the identified mutations were highly conserved in different species. Interestingly, the conservation could be partially observed in nebulette itself. The mutations at codons 581 and 652 showed this conservation also in the different nebulin-like repeats (Figure $2 \mathrm{~A}$ ). Furthermore, the changes were non-conservative; namely, they changed the properties of the residues such as polarity or size, indicating deleterious effects on protein structure.

The localization of the mutations in a functional protein domain underscores a possible pathogenic effect. Notably, five of the identified mutations reside in the nebulin-like repeats, the basic functional units for actin binding. The sixth mutation (p.P916L) is located in the serine-rich linker domain responsible for cellular targeting in the Z-disk (as shown by Panaviene et al. [23]). The fact that transgenic animal models expressing human NEBL mutations generated by Purevjav et al. resembled a cardiomyopathic phenotype strongly enhances the confidence in our results [13]. Finally, the $N E B L$ gene that encodes the Z-disk protein, nebulette, is an excellent candidate gene to contribute to heart muscle disease. The fact that NEBL is preferentially expressed in the heart makes such speculation more plausible. Furthermore, there are a number of known cardiomyopathy disease genes encoding Z-disk proteins (Figure 4). This scheme is quite fitting for nebulette.

Purevjav et al. [13] drew attention to four missense mutations in NEBL (p.K60N, p.Q128R, p.G202R, p.A592E). These are also located in the nebulin-like repeats and are in close vicinity to five mutations identified by us affecting the same repeat domain in two cases (Figure $1 \mathrm{~B}$ ). They found $N E B L$ mutations in two newborn DCM patients (one with endocardial fibroelastosis) and two patients with DCM manifestation in adulthood [13]. We found mutations in familial as well as sporad-

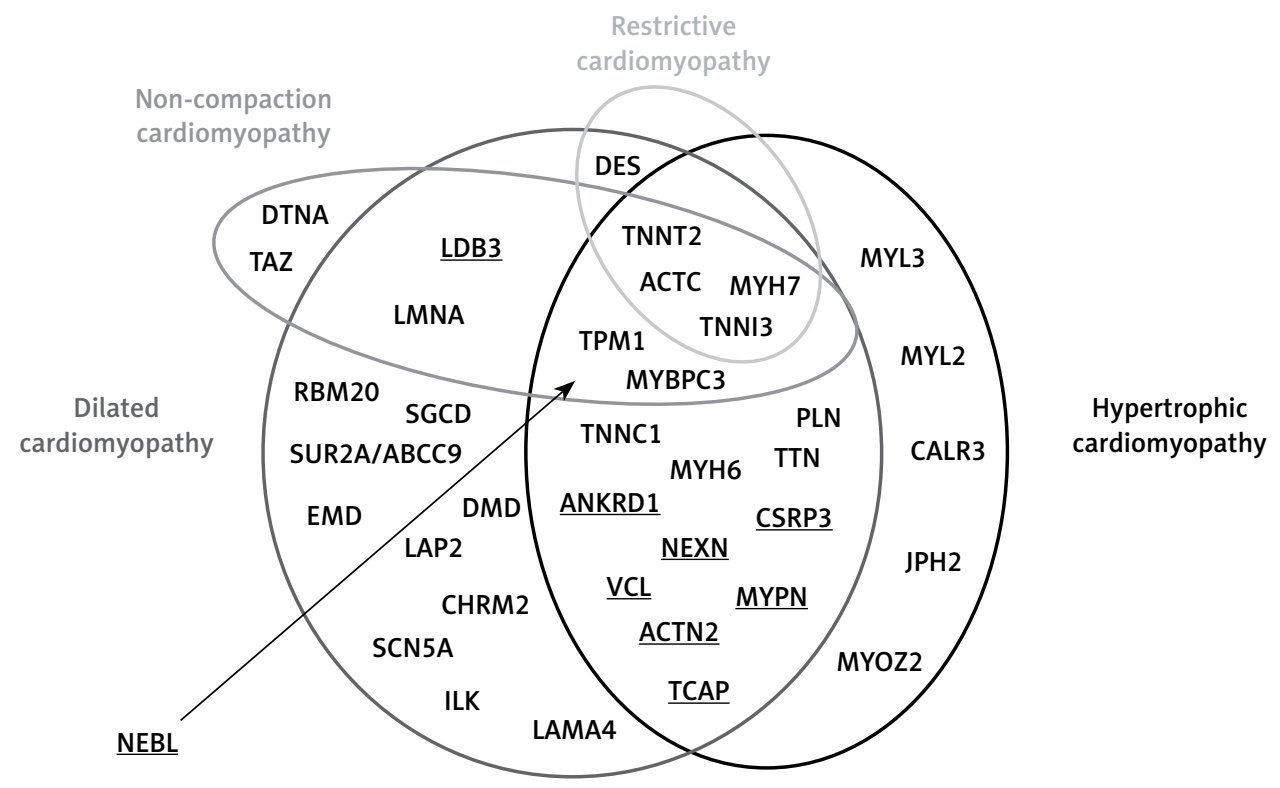

Figure 4. Diagram of the overlap of disease genes causing four different cardiomyopathy forms. Thirty-seven genes have been described so far, of which 20 genes cause at least two different forms of cardiomyopathy. Underlined gene symbols encode Z-disk proteins. Of note, all seven Z-disk genes are involved in the pathogenesis of two different cardiomyopathy forms. Conclusively, we have described the Z-disk gene NEBL as possibly associated with HCM, DCM and LVNC in this study (data according to [1-4]) 
ic cases with DCM and additionally in cases with HCM or LVNC. Furthermore, we identified clinical manifestation of cardiomyopathy mostly in adulthood (the exception was one child with severe HCM). There seems to be a possible relation to age as seen in the probands with NEBL mutations who have an age at diagnosis between 41 and 53 years (Table II).

Relatives without cardiomyopathy often showed a negative genotype, whereas pathological phenotypes co-segregated with an NEBL mutation (Figure 3). However, two individuals, C: III-2, 19 years old, and C: III-3, 23 years old, from family C were mutation carriers without a cardiomyopathy phenotype. This could be attributed to their young age, which is well known to be associated with a low probability of cardiomyopathy expression due to age-related penetrance $[1,4]$. The unaffected individual A: II-5, a mutation carrier and 56 years old, may be explained by the fact that penetrance increases with age but remains less than $100 \%$, as Watkins et al. stated in their review [4]. The clinical significance of the p.1652L variant in family $B$ is puzzling. The mother (B: I-2) showing hypertrophy and aortic stenosis was a carrier of the mutation. However, two relatives with some degree of hypertrophy did not carry the mutation. While individual B: I-1 had an unequivocal HCM, individual B: II-2 showed mild hypertrophy and arterial hypertension and therefore may be regarded as a likely phenocopy (i.e. masquerading as HCM). On the one hand, p.1652L is highly conserved in orthologs and NLRs and quite rare in patients and controls ( $0.01 \%$ according to ESP). On the other hand, it is the only conserved change predicted to be benign by PolyPhen 2 and has an allelic frequency of $0.3 \%$ according to dbSNP. Taken together, we would classify p.1652L as a variant of unknown significance.

We suggest that NEBL mutations may generate cardiomyopathy phenotypes. Thus we must propose mechanisms. Moncman and Wang showed that a targeted disruption of nebulette expression in cardiomyocytes significantly altered myofibril assembly and function [24]. Purevjav et al. validated the functional effects of four human NEBL missense mutations in detail [13]. They generated transgenic mice with cardiac-restricted overexpression of human wild-type and mutant nebulette, showing a variety of phenotypes and severity in vivo ranging from embryonic lethality due to cardiac abnormalities to LV enlargement and diminished LV function at later stages [13]. Effects at the molecular/cellular level were changes in I-band and Z-disk composition and abnormal distribution of mutant nebulette under mechanical stretch. Their findings showed that $N E B L$ mutations have strong but different effects on nebulette function itself and the function of interacting proteins depending on the location of the respective mutation. Ram and Blaxall concluded that nebulette may play dual roles as both a structural protein of the Z-disk, and also - possibly through its binding partners - as a regulatory protein of signaling pathways [25]. The findings of Purevjav et al. suggest that nebulette is required for normal sarcomere genesis and Z-disk stabilization during development and adult function [13].

We were struck by the fact that nearly all Z-disk genes were associated with DCM as well as HCM, as Bos and Ackerman noted [26] (underlined genes in Figure 4). They pointed out that for Z-disk-mediated cardiomyopathies it is difficult to envision how divergent phenotypes emerge, especially because maladaptive dilatation seems a more plausible phenotypic response than maladaptive hypertrophy, given the role of the Z-disk as a sarcomeric stretch sensor and mechanoreceptor [26]. The divergence in phenotypes may reflect the multiple roles of nebulette in modulating cardiomyocyte stretch-strain with specific structural/cytoskeletal modifications, as Ram and Blaxall proposed [25]. However, there is still a wide knowledge gap between the specific genetic cause and the mechanism producing a specific clinical phenotype [3]. A complete understanding of how underlying mutation and resulting phenotype are connected is still pending for the cardiomyopathies. It is obvious that the ultimate phenotype represents the sum of all the parts: genotype, mutant RNA and protein expression, further genetic variants, and epigenetic influences as well as environmental factors [3]. But we still lack integrated approaches that could elucidate the mechanisms.

There may be some caveats to the interpretation of the present study. First, it is limited by the fact that the families were too small to perform linkage analysis. Cosegregation analysis was possible in four families and produced inconclusive results in one of them. Second, no functional studies were performed. We were therefore cautious with the assignment of true disease causation [2, $21,27,28]$. However, considering the conservation of the affected amino acid, the nature and location of the change, its absence in controls and the general population, and the reported animal models of related mutations point to a possible significance of five of the identified mutations (as we regard p.1652L as a variant of unknown significance).

It was not possible to screen all known genes associated with cardiomyopathy; so we cannot exclude the possibility that the mutation carriers may be double heterozygotes. This is in particular true for DCM genes, whereas we excluded nearly all HCM genes. However, even greater patient 
numbers may be necessary to draw more general conclusions about the frequency of NEBL mutations in cardiomyopathy patients. Our purpose here was to draw attention to $N E B L$, a gene that we believe warrants much more multidisciplinary research attention.

In conclusion, our data strongly suggest that five different NEBL mutations are possibly associated with various cardiomyopathies. Already known for DCM, we herein describe the first possible NEBL mutations in HCM and LVNC. Our findings underline the notion that the cardiomyopathies are true allelic diseases. With regard to next generation sequencing methods, NEBL may be added to a set of cardiomyopathy genes which now can be quickly analyzed in parallel with a target enrichment approach.

\section{Supplemental data}

Supplemental data contain Supplemental methods, Supplemental results, Supplemental Table SI and Supplemental Figures S1, S2, and S3.

\section{Acknowledgments}

This study was supported by a Charité research grant, Assistance Publique - Hôpitaux de Paris (PHRC programme hospitalier de recherche clinique AOM95082), the CONNY-MAEVA charitable foundation, the Leducq Foundation (Fondation Leducq) (Eurogene Heart Failure network), a European Union FP7 grant (INHERITANCE network), the Italian Ministry of University and Research (grant 20083EWHYR_002), and the Czech Ministry of Health (project for conceptual development of research organization 00064203). We are grateful to the patients and their relatives for their participation in the study. We thank Andrea Behm, Uzma Aslam, Laetitia Dubosq-Bidot, and Peng Xu for excellent technical support.

\section{Conflict of interest}

The authors declare no conflict of interest.

\section{References}

1. Richard P, Villard E, Charron P, Isnard R. The genetic basis of cardiomyopathies. J Am Coll Cardiol 2006; 48: A79-89.

2. Hershberger RE, Cowan J, Morales A, Siegfried JD. Progress with genetic cardiomyopathies: screening, counseling, and testing in dilated, hypertrophic, and arrhythmogenic right ventricular dysplasia/cardiomyopathy. Circ Heart Fail 2009; 2: 253-61.

3. Perrot A, Dietz R, Osterziel KJ. Is there a common genetic basis for all familial cardiomyopathies? Eur J Heart Failure 2007; 9: 4-6.

4. Watkins H, Ashrafian H, Redwood C. Inherited cardiomyopathies. N Engl J Med 2011; 364: 1643-56.

5. Duboscq-Bidot L, Xu P, Charron P, et al. Mutations in the $Z$ band protein myopalladin gene and idiopathic dilated cardiomyopathy. Cardiovasc Res 2008; 77: 118-25.
6. Moncman CL, Wang K. Nebulette: a 107 kD nebulin-like protein in cardiac muscle. Cell Motil Cytoskeleton 1995; 32: 205-25.

7. Esham M, Bryan K, Milnes J, Holmes WB, Moncman CL. Expression of nebulette during early cardiac development. Cell Motil Cytoskeleton 2007; 64: 258-73.

8. Moncman CL, Wang K. Functional dissection of nebulette demonstrates actin binding of nebulin-like repeats and Z-line targeting of SH3 and linker domains. Cell Motil Cytoskeleton 1999; 44: 1-22.

9. Holmes WB, Moncman CL. Nebulette interacts with filamin C. Cell Motil Cytoskeleton 2008; 65: 130-42.

10. Bang ML, Mudry RE, McElhinny AS, et al. Myopalladin, a novel 145-kilodalton sarcomeric protein with multiple roles in Z-disc and I-band protein assemblies. J Cell Biol 2001; 153: 413-27.

11. Ogut $\mathrm{O}$, Hossain MM, Jin JP. Interactions between nebulin-like motifs and thin filament regulatory proteins. J Biol Chem 2003; 278: 3089-97.

12. Bonzo JR, Norris AA, Esham M, Moncman CL. The nebulette repeat domain is necessary for proper maintenance of tropomyosin with the cardiac sarcomere. Exp Cell Res 2008; 314: 3519-30.

13. Purevjav E, Varela J, Morgado M, et al. Nebulette mutations are associated with dilated cardiomyopathy and endocardial fibroelastosis. J Am Coll Cardiol 2010; 56: 1493-502.

14. Richard P, Charron P, Carrier L, et al. Hypertrophic cardiomyopathy: distribution of disease genes, spectrum of mutations, and implications for a molecular diagnostic strategy. Circulation 2003; 107: 2227-32.

15. Perrot A, Schmidt-Traub H, Hoffmann B, et al. Prevalence of cardiac beta-myosin heavy chain gene mutations in patients with hypertrophic cardiomyopathy. J Mol Med 2005; 83: 468-77.

16. Perrot A, Sigusch HH, Nägele $H$, et al. Genetic and phenotypic analysis of dilated cardiomyopathy with conduction system disease: demand for strategies in the management of presymptomatic lamin A/C mutant carriers. Eur J Heart Failure 2006; 8: 484-93.

17. Mestroni L, Maisch B, McKenna WJ, et al. Guidelines for the study of familial dilated cardiomyopathies. Eur Heart J 1999; 20: 93-102.

18. Petersen SE, Selvanayagam JB, Wiesmann F, et al. Left ventricular non-compaction: insights from cardiovascular magnetic resonance imaging. J Am Coll Cardiol 2005; 46: 101-5.

19. Arimura T, Nakamura T, Hiroi S, et al. Characterization of the human nebulette gene: a polymorphism in an actin-binding motif is associated with nonfamilial idiopathic dilated cardiomyopathy. Hum Genet 2000; 107: 440-51.

20. Fokstuen S, Lyle R, Munoz A, et al. A DNA resequencing array for pathogenic mutation detection in hypertrophic cardiomyopathy. Hum Mutat 2008; 29: 879-85.

21. Norton N, Robertson PD, Rieder MJ, et al. Evaluating pathogenicity of rare variants from dilated cardiomyopathy in the exome era. Circ Cardiovasc Genet 2012; 5: 167-74.

22. Vitkup D, Sander C, Church GM. The amino acid mutational spectrum of human genetic disease. Genome Biol 2003; 4: R72.

23. Panaviene Z, Moncman CL. Linker region of nebulin family members plays an important role in targeting these molecules to cellular structures. Cell Tissue Res 2007; 327: 355-69. 
24. Moncman CL, Wang K. Targeted disruption of nebulette protein expression alters cardiac myofibril assembly and function. Exp Cell Res 2002; 273: 204-18.

25. Ram R, Blaxall BC. Nebulette mutations in cardiac remodeling: big effects from a small mechanosensor. J Am Coll Cardiol 2010; 56: 1503-5.

26. Bos JM, Ackerman MJ. Z-disc genes in hypertrophic cardiomyopathy. J Am Coll Cardiol 2010; 55: 1136-8.

27. Saracyn M, Płoski R, Niemczyk S. Contemporary role of medical genetics in internal medicine. Arch Med Sci 2013; 9: 594-600.

28. Houshmand M, Montazeri M, Kuchekian N, Noohi F, Nozar G, Zamani A. Is 8860 variation a rare polymorphism or associated as a secondary effect in HCM disease? Arch Med Sci 2011; 7: 242-6.

29. Moncman CL, Wang K. Architecture of the thin filament-Z-line junction: lessons from nebulette and nebulin homologies. J Muscle Res Cell Motil 2000; 21: 153-69.

30. Millevoi S, Trombitas K, Kolmerer B, et al. Characterization of nebulette and nebulin and emerging concepts of their roles for vertebrate Z-discs. J Mol Biol 1998; 282: 111-23. 


\section{Supplemental methods}

\section{Patients}

In total, 389 unrelated cardiomyopathy patients (HCM, DCM, or LVNC) were evaluated according to the American Heart Association definitions [1]. They were all of European origin (with the exception of the French DCM cohort with 93\% Western Europeans). All cohorts were examined using a comparable protocol based on the medical history, physical examination, two-dimensional and M-mode echocardiography, and 12-lead electrocardiogram (ECG), without knowledge of the genetic status. Holter ECG, heart catheterization, angiography, and cardiac magnetic resonance imaging (MRI) were performed in some patients as clinically indicated. Causes of heart disease such as coronary artery disease, myocarditis, severe arterial hypertension, and valvular heart disease were carefully excluded from the present study. All subjects agreed to participate in the study and gave written informed consent. The study was approved by the responsible local institutional review boards in the respective centers.

The diagnosis of HCM was made according to the established criteria. Briefly, the major inclusion criterion was the presence of interventricular septal thickness (IVS) $\geq 13 \mathrm{~mm}$ (15 mm if sporadic, $13 \mathrm{~mm}$ if familial) in the absence of other known causes of hypertrophy (such as hypertension and aortic stenosis) often accompanied by major ECG abnormalities such as negative T-waves, pathological Q-waves, and left ventricular hypertrophy.

Diagnosis of DCM was made according to the established criteria. The main criteria for DCM were defined as an echocardiographically measured left ventricular ejection fraction (EF) $<45 \%$ and left ventricular end-diastolic diameter (LVEDD) $>56 \mathrm{~mm}$ (or greater than the theoretical value according to age and body surface) in the absence of coronary artery disease. The patients had no systemic hypertension. Familial DCM was diagnosed if another affected relative had proven or highly suspected DCM according to the European guidelines of Mestroni et al. [2].

Diagnosis of LVNC was based on established criteria. All included patients showed a thickened LV myocardial wall with a two-layered structure comprising a compact (C) epicardial layer and a noncompacted (NC) endocardial layer of prominent trabeculations and deep intertrabecular recesses. The maximal end-diastolic ratio of noncompacted to compacted wall was > 2.3 measured by MRI. The patients had no systemic hypertension.

We used two established control cohorts [3]. The control cohort A comprised 320 Caucasian sex-matched unrelated individuals without cardiomyopathy (who had preserved EF > 55\% and
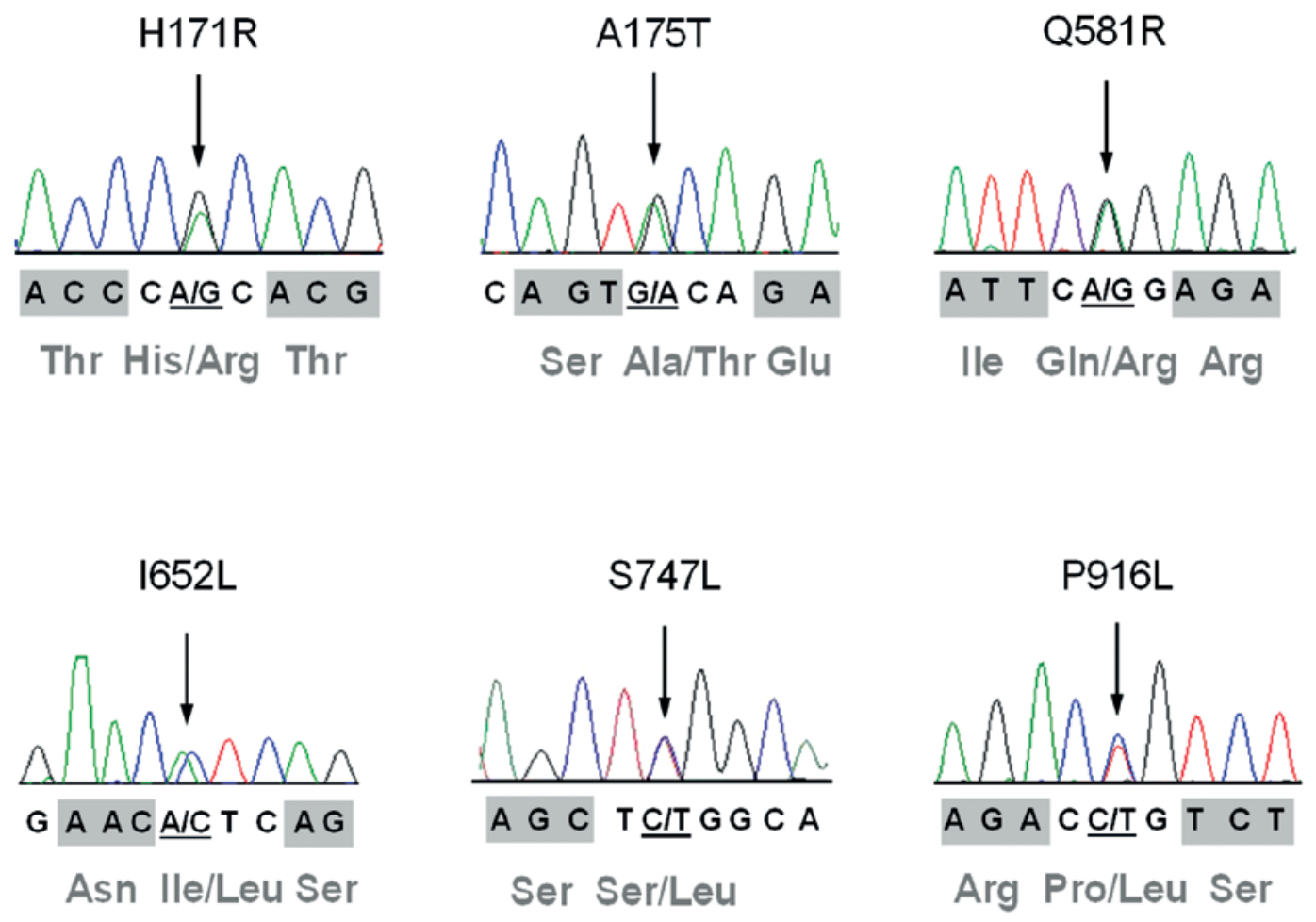

Supplemental Figure S1. DNA sequencing electropherograms demonstrating heterozygosity for the six detected $N E B L$ mutations. The missense mutations are shown as two overlapping peaks (marked with an arrow). Codons are marked with gray blocks 
no ventricular dilatation and hypertrophy as shown by echocardiography and ventriculography and/or angiography). All of these individuals were older than 50 years (in order to exclude late onset cardiomyopathy). The second control cohort B included 192 Caucasian sex-matched blood donors from the local blood transfusion service without heart disease. None of the control individuals was hypertensive.

\section{Genetic analysis}

A bioinformatic approach for detected variants was used which was based on presence or absence of these in population-based databases. First, we searched dbSNP (build 135, at http:// www.ncbi.nlm.nih.gov/snp), which also includes data from the 1000 Genomes project. Second, we searched the Exome Variant Server (EVS) provided by the Exome Sequencing Project (ESP) of the National Heart, Lung and Blood Institute (http:// evs.gs.washington.edu/EVS/, Tennessen et al. [4]). The EVS data release ESP5400 is taken from 5379 samples drawn from multiple ESP cohorts and represents the first data freeze of the ESP exome variant data (EVS release version: v.0.0.12). The ESP dataset includes exome data for 3510 individuals of European descent, which matched our patients. Third, we performed mutation prediction by the two most widely used analysis tools to predict the possible impact of an amino acid substitution. PolyPhen 2 analysis was performed through the portal http://genetics.bwh. harvard.edu/pph2/ [5]. Mutation Taster analysis was performed through the portal http://www. mutationtaster.org [6].

\section{Supplemental results}

\section{Clinical features}

Proband II-3 of family B, carrier of mutation p.1652L, had severe hypertrophy (IVS $32 \mathrm{~mm}$, PWT $16 \mathrm{~mm}$ ) which progressed into thinning of the septum (IVS $26 \mathrm{~mm}$ ), followed by dilatation (LVEDD from 43 to $62 \mathrm{~mm}$ ) and reduction of EF (from $58 \%$ to $26 \%$ ) over a period of about 20 years. Therefore, she underwent heart transplantation at the age of
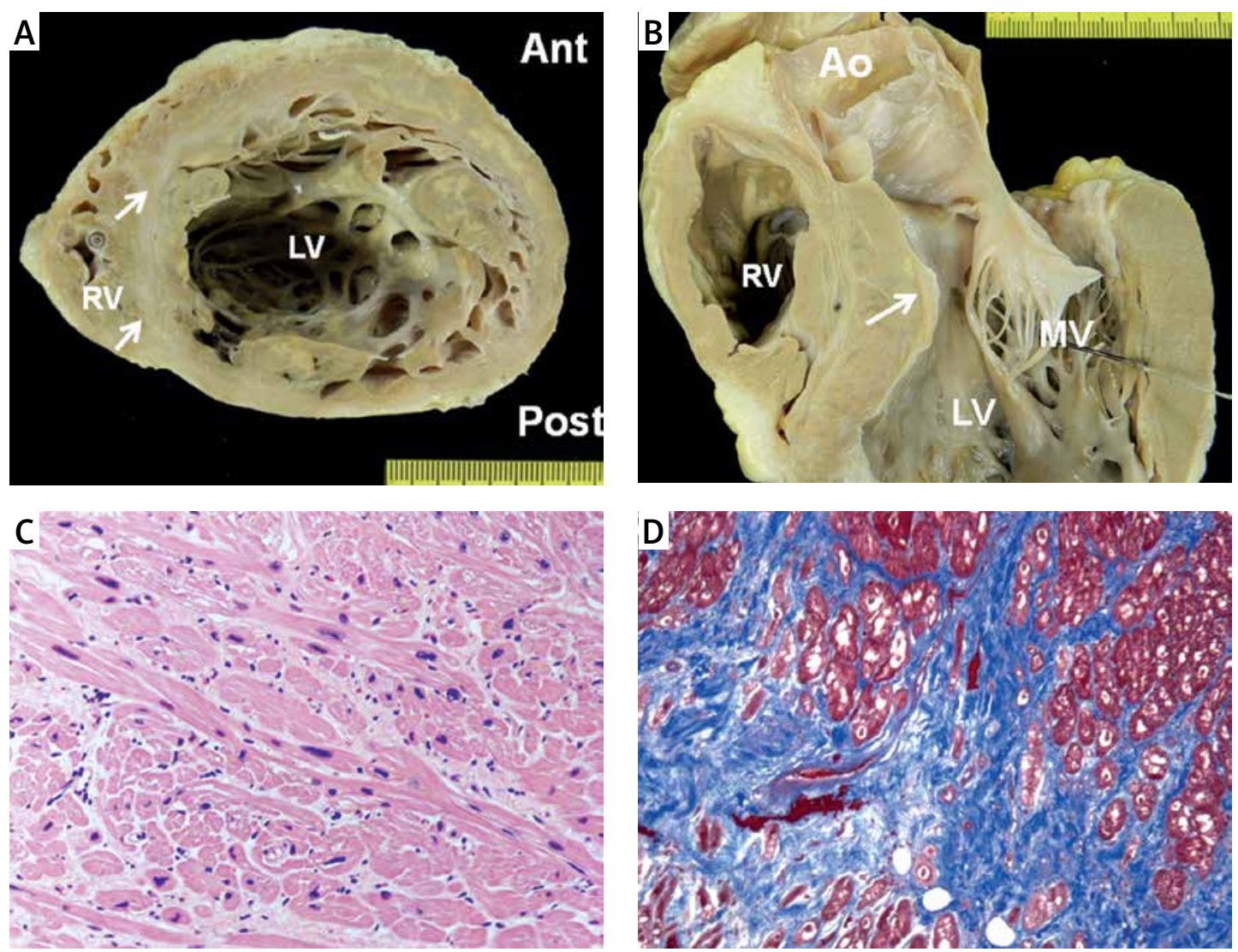

Supplemental Figure S2. Heart removed at transplantation of a HCM patient with NEBL mutation (proband B: II-2 of family B). A - Apical transverse section of the heart showing dilatation of the left ventricular (LV) and thinning of the septum and ventricular free wall; whitish areas (arrows) are indicative of septal scarring. B - Long-axis LV section (atria missing due to transplantation). Subaortic outflow tract obstruction with endocardial fibrous plaque (arrow). C - Histology of right ventricle (RV) showing myocyte hypertrophy and disarray; hematoxylin eosin stain, 160x. D - Histology of the septum with extensive replacement fibrosis and myocyte vacuolization; Azan-Mallory stain 200x 
A. Perrot, P. Tomasov, E. Villard, R. Faludi, P. Melacini, J. Lossie, N. Lohmann, P. Richard, M. De Bortoli, A. Angelini, A. Varga-Szemes, S.R. Sperling, T. Simor, J. Veselka, C. Özcelik, P. Charron

Supplemental Table SI. Detected variants in the coding region of NEBL

\begin{tabular}{|c|c|c|c|c|c|c|}
\hline DNA change & AA change & Exon & Variant & Known/novel & dbSNP ID & Reference \\
\hline c.109T>C & p.L37L & 2 & sSNP & Known & rs140734883 & - \\
\hline C. $180 G>C$ & p.K60N & 3 & nsSNP & Known & rs41277374 & Purevjav et al. (2010) \\
\hline c. $191 \mathrm{~A}>\mathrm{G}$ & p.K64R & 3 & nsSNP & Known & rs71578975 & - \\
\hline c. $489 \mathrm{~T}>\mathrm{C}$ & p.Y163Y & 6 & sSNP & Known & rs141153708 & - \\
\hline c. $604 \mathrm{G}>\mathrm{A}$ & p.G202R & 7 & nsSNP & Known & rs137973321 & Purevjav et al. (2010) \\
\hline c. $1051 \mathrm{~A}>\mathrm{G}$ & p.M351V & 11 & nsSNP & Known & rs4025981 & Arimura et al. (2000) \\
\hline c. $1132 G>C$ & p.D378H & 12 & nsSNP & Known & rs41277370 & - \\
\hline C. $1672 A>C$ & p.R558R & 17 & sSNP & Novel & - & - \\
\hline c. $1861 \mathrm{~A}>\mathrm{G}$ & p.1621V & 18 & nsSNP & Known & rs79718972 & - \\
\hline c. $1962 T>A$ & p.N654K & 19 & nsSNP & Known & rs4748728 & Arimura et al. (2000) \\
\hline c. $2031 \mathrm{G}>\mathrm{A}$ & p.R677R & 20 & SSNP & Known & rs1006363 & - \\
\hline c. $2182 A>G$ & p.T728A & 22 & nsSNP & Known & rs71535732 & Arimura et al. (2000) \\
\hline c. $2654 C>T$ & p.S885F & 26 & nsSNP & Known & rs143584663 & - \\
\hline c. $2997 A>G$ & р.Т999Т & 28 & sSNP & Known & rs2296614 & - \\
\hline
\end{tabular}

$A A$ - amino acid, sSNP - synonymous SNP, nSSNP - non-synonymous SNP, dbSNPID - SNP database (build 135) identifier.

46 years. This "end-stage" HCM patient showed LV chamber dilatation with diffuse transmural fibrous replacement in the ventricular septum and LV free wall (Supplemental Figures S2A and S2B). Histopathological features of HCM such as myocyte hypertrophy and myocyte disarray were present in her heart (Supplemental Figures S2C) as well as extensive replacement fibrosis and myocyte vacuolization (Supporting Figure S2D).

Cardiac MRI of proband II-1 of family G, carrier of mutation p.P916L, revealed LVNC affecting the entire apical region as well as the mid-segments of the lateral and anterolateral wall (7 segments in 17-segment model) (Supplemental Figure S3). The maximal NC/C ratio was 3.8. The left ventricle was severely dilated (LVEDD $66 \mathrm{~mm}$, LVESD $53 \mathrm{~mm}$ ), with severely reduced EF (30\%). Due to multiple episodes of non-sustained ventricular tachycardia, an ICD was implanted at the age of 43 years.

\section{Further detected variants in NEBL}

We identified 14 single nucleotide variants including five synonymous and nine non-synonymous variants (Supplemental Table SI). All of these were detected in the cardiomyopathy cohorts in different frequencies (from 1\% to $31 \%$ ) and were represented in dbSNP except for one. Two of these variants, p.K60N and p.G202R, were recently described by Purevjav et al. in two adult DCM patients [7]. To validate our results, we analyzed a second independent control group (cohort
B) in addition to control cohort A (1024 control alleles in total). We detected the variant p.K60N in five HCM patients (frequency $2.3 \%$ ). Subsequent evaluation in the two control cohorts $A$ and $B$ revealed p.K60N in 14 subjects (frequency 2.7\%) including one with homozygous genotype. The variant p.G202R was identified in one $\mathrm{HCM}$ patient (frequency $0.5 \%$ ). Analysis of the two control cohorts revealed four individuals carrying the heterozygous variant (frequency $0.8 \%$ ). A search of the ESP data showed that p.K60N and p.G202R are present in allele frequencies of $0.31 \%$ and $1.08 \%$, respectively.

\section{Supplemental references}

1. Maron BJ, Towbin JA, Thiene G, et al. Contemporary definitions and classification of the cardiomyopathies: an American Heart Association Scientific Statement from the Council on Clinical Cardiology, Heart Failure and Transplantation Committee; Quality of Care and Outcomes Research and Functional Genomics and Translational Biology Interdisciplinary Working Groups; and Council on Epidemiology and Prevention. Circulation 2006; 113: 1807-16.

2. Mestroni L, Maisch B, McKenna WJ, et al. Guidelines for the study of familial dilated cardiomyopathies. Eur Heart J 1999; 20: 93-102.

3. Posch MG, Posch MJ, Geier C, et al. A missense variant in desmoglein-2 predisposes to dilated cardiomyopathy. Mol Genet Metabol 2008; 95: 74-80.

4. Tennessen JA, Bigham AW, O'Connor TD, et al. Evolution and functional impact of rare coding variation from deep sequencing of human exomes. Science 2012; 337: 64-9. 

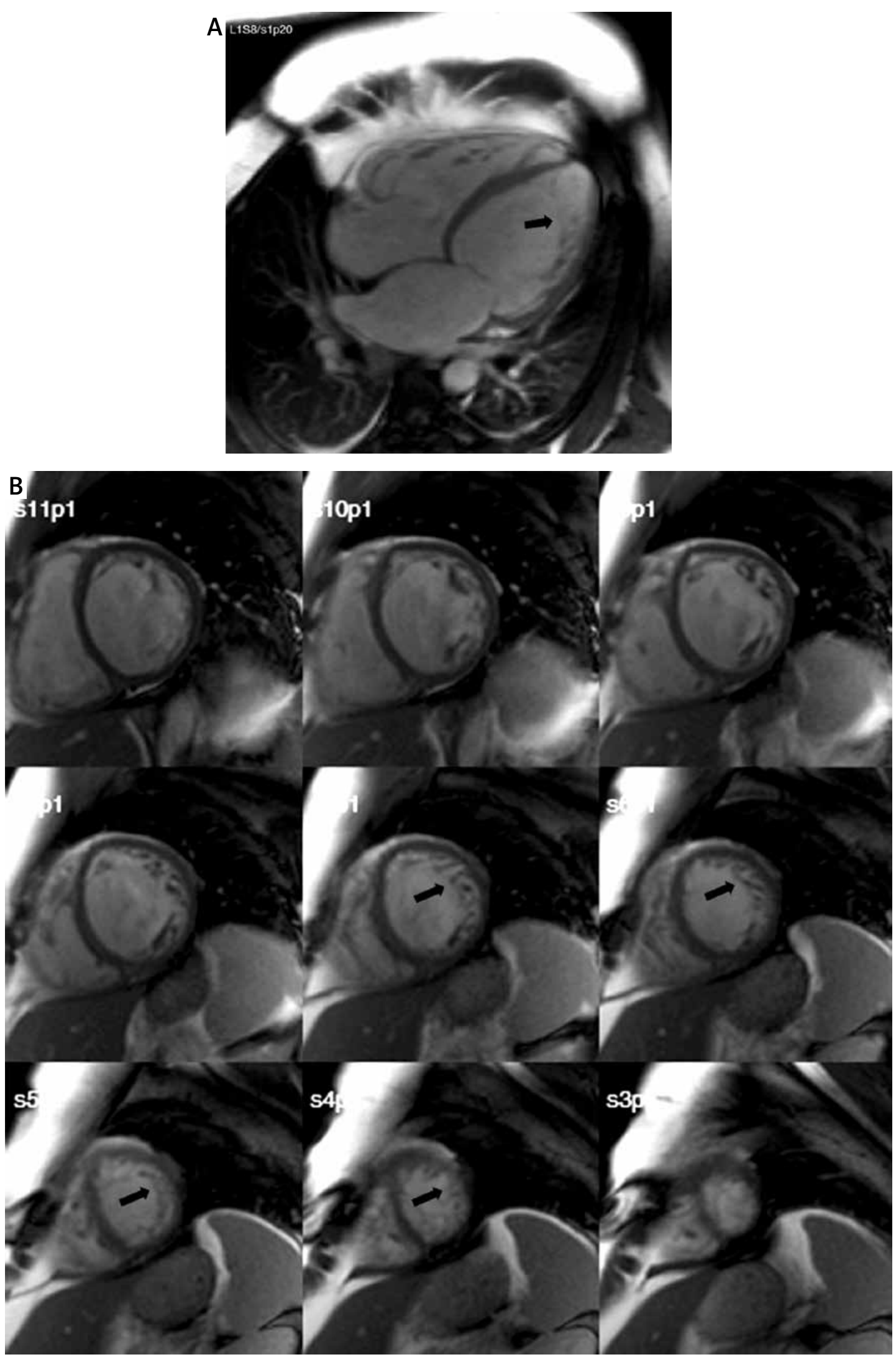

Supplemental Figure S3. Magnetic resonance imaging of the heart of the LVNC patient with NEBL mutation (proband G:II-1 of family G). ECG retrogated SSFP movie sequence (trufi) was used to assess ventricular function on a 1.5T Siemens Avanto instrument. A - Diastolic four-chamber view shows significant LVNC (arrow) affecting the entire apical region as well as the mid-segment of the lateral wall. B - Short axis images show significant LVNC (arrows) affecting the entire apical region as well as the mid-segments of the lateral and anterolateral wall 
A. Perrot, P. Tomasov, E. Villard, R. Faludi, P. Melacini, J. Lossie, N. Lohmann, P. Richard, M. De Bortoli, A. Angelini, A. Varga-Szemes, S.R. Sperling, T. Simor, J. Veselka, C. Özcelik, P. Charron

5. Adzhubei IA, Schmidt S, Peshkin L, et al. A method and server for predicting damaging missense mutations. Nat Methods 2010; 7: 248-9.

6. Schwarz JM, Rödelsperger C, Schuelke M, Seelow D. MutationTaster evaluates disease-causing potential of sequence alterations. Nat Methods 2010; 7: 575-6.

7. Purevjav E, Varela J, Morgado $M$, et al. Nebulette mutations are associated with dilated cardiomyopathy and endocardial fibroelastosis. J Am Coll Cardiol 2010; 56: 1493-502. 